\title{
ARE INCUBATOR SCALES ACCURATE FOR WEIGHING VENTILATED BABIES? A PILOT STUDY ON 'VENTILATED' MANIKINS
}

\author{
U. El-Kafrawy
}

NNU, Salford Royal Hospital, Manchester, UK

Background: Accurate weight measurement of ventilated babies facilitates optimum care. Incubator scales may be used to weigh ventilated babies, rather than risk destabilisation by disconnection. In a survey of tertiary unit neonatologists, $49 \%$ believe incubator scales to be accurate for trend weights, $38 \%$ believe them to be accurate for individual weights.

\section{Aims:}

1.To ascertain whether incubator scales are accurate for weighing ventilated manikins.

2. If inaccurate, develop a method to correct the inaccuracy.

\section{Methodology:}

1. Intubated manikins of various weights connected to ventilator tubing were weighed on incubator scales and then disconnected and weighed on standalone scales.

2. A device attachable to ventilator tubing was developed. 60 manikins, 30 attached to oscillator tubing and 30 attached to conventional ventilator tubing, were weighed on incubator scales with and without the device and then on standalone scales.

\section{Results:}

1. Large discrepancy found between manikin weights on incubator and standalone scales.

2. Data was analysed using Stats direct, and the Bland Altman method.

In oscillated manikins, mean error in weight was $42.2 \mathrm{~g}$ (SD of $8 \mathrm{~g}$ ). With the device attached mean error $1.2 \mathrm{~g}$ (SD 3.9g). In ventilated manikins, mean error in weight was $32.7 \mathrm{~g}$ (SD of $3.5 \mathrm{~g}$ ). With the device attached mean error 9.2g (SD 2.3g). An F-test for variance showed the improvement in accuracy was $(\mathrm{p}<0.0001)$ for the former and $\mathrm{p}=0.01$ for the latter.

Conclusion: Results indicate that incubator scales are inaccurate for trend or individual weights in ventilated manikins and that an attachable device can be used to correct the inaccuracy. 